\title{
Design of a Node for an Underwater Sensor Network
}

\author{
Abraham Varughese, \\ NSTL \\ Visakahaptnam \\ India
}

\begin{abstract}
The application Sensor Network has emerged as an amalgamation of the two areas namely sensors and communication systems. Sensor network consists of geographically distributed sensors which monitors physical or environmental conditions and cooperatively passes their data through the network to a main location. The applications of Sensor Networks are vivid and varied. It has revolutionized the area of measurement and networking. Underwater Sensor Networks consist of a number of sensors that are deployed to perform collaborative monitoring in water. Important constituent of an underwater network is a network node. Here we discuss the development of an underwater node.
\end{abstract}

Keywords

Acoustic, Network, Node, AUV, Embedded, Protocols, Sensor

\section{INTRODUCTION}

Underwater Man has forayed into all parts of the planet earth with his quest for conquering new avenues. He has explored great heights and all continents. Although man has walked on the moon and seen the surface of Mars, man has explored less than $1 \%$ of the earth's sea floor with his own eyes. Thus, the major chunk of this sphere is rather unexplored especially the underwater scenario. Compared to man's familiarity with land, there are still many unexplored areas underwater.

Sensor network consists of sensors which are interconnected through either wired or wireless means. Networking is an important constituent which makes the geographic separation of the nodes possible and the nodes can be placed in the area of interest. Further effective inter node communication is required for the data aggregation from different nodes. An Underwater Sensor Network (UWSN) is an emerging network paradigm which consists of a number of underwater sensor nodes and Autonomous Underwater Vehicles (AUVs) that are deployed to perform collaborative monitoring and resource exploration tasks over a given area. Underwater systems such as remote sensors, autonomous underwater vehicles and control vessels are indispensible in underwater research and communication. Traditional data acquisitions systems in underwater area work on the principle of recording and offline analysis. The recording instruments will be deployed, data acquisition will be carried out and then instruments will be recovered after a specific period. Data will be retrieved from the system and analysis will be carried out. Hence there is no scope of online analysis and decision making.

As electromagnetic waves get rapidly attenuated, acoustic communication is employed in underwater scenario. Sound waves being much slower than electromagnetic waves, the

\author{
Seetharamaiah.P \\ Andhra University \\ Visakahaptnam \\ India
}

\begin{abstract}
latency in underwater communication is characteristically much higher than in their terrestrial counterparts [1]. Due to the limitations like multi-path propagation and ambient noise, it will be possible to achieve only very slow data rates. Moreover, data errors and packet loss rate is by and large much higher. Various aspects of a protocol need to be decided and formulated in order to achieve a fully efficient and purposeful network. Issues to be addressed are modulation, synchronization, packet formatting, error correction, medium access control, addressing, routing, etc. Absence of specific well defined and unified framework is still an issue in the research of underwater acoustics and underwater networks[3]. There are several independently developed techniques and schemes related to these areas. Integration of these techniques and amalgamation to a framework will hasten the development of underwater network research. A framework becomes established only when it has got a wide acceptance. For this, a framework needs to take into consideration a wide variety of diverse constraints and requirements for various underwater applications.
\end{abstract}

\section{APPLICATIONS OF UNDERWATER SENSOR NETWORKS}

There are a wide variety of applications for Underwater Sensor Networks (UWSNs) and many more can be thought of. Its wide utility is giving it noticeable importance. The major applications come under the gamete of both military and commercial purposes and considerable research is going on in various spheres aiming at a range of applications. More and more research interest and efforts are shifting to this area in recent years. The applications of Underwater sensor nodes can be bucketed under oceanographic data collection, pollution monitoring, offshore exploration, disaster prevention, assisted navigation and tactical surveillance applications. [1]

Underwater networks can play a key role in ocean surveillance. It has been reported that a shallow water acoustic network has been implemented to enable cooperative mine counter measures [5]. The Achilles heel to the security of a country along a coast is its coastline. With greater security threats through the sea, offshore security monitoring has gained utmost importance. Offshore security monitoring emerged as one of the key areas in recent times because of the prevailing global security situation and the threat coming through the seafront. It is very difficult to have a manned surveillance for the coastal line. So the alternative is to have an automated surveillance system. The essential ingredient of such a network is sensors. These sensors are to be interconnected to take a decision.

Underwater Sensor Networks can be used to monitor the surrounding activities and external intrusion by gauging the acoustic noise of the vehicle engine or short range detection using laser. An acoustic sensor will measure the 
passive noise of the intruding vehicle. The detection system consists of the acoustic sensor, pre amplifier, Analog to Digital converter and Digital signal processor to detect and classify the target.

The applications of the UWSN can be summarized as:

- $\quad$ Protection of installations in the sea coast

- Protection of offshore installations like oil rigs

- Monitoring of coastal guard forces

- Co ordination of anti-smuggling operations and anti terrorist activities.

- Conducting a reconnaissance mission.

- Conducting tactical surveillance

\section{DIFFERENCES BETWEEN TERRESTRIAL AND UNDER WATER SENSOR NETWORKS}

Underwater sensor networks are a new research topic and there are many unsolved issues. The unique underwater environment is the root cause of these issues. An underwater acoustic channel is different from a groundbased radio channel in many aspects. The main differences between terrestrial and underwater sensor networks are in terms of cost, power, limited storage capacity, deployment challenges, sensor failures, mechanical construction, limitations of acoustic channel and dependence on climatic conditions.

There are many design challenges in establishing an underwater acoustic network, particularly a collaborative network with both static and dynamic nodes. Underwater networks are difficult to realize unlike their terrestrial counterparts. Terrestrial wireless sensor network technologies are already mature and are being deployed for various data collection. In the case of underwater sensor networks, protocols which are relevant in underwater scenario are to be decided.

\subsection{Acoustic communication}

Underwater networks have to have a good communication medium. The options available are electromagnetic propagation or acoustic propagation. In terrestrial communication, electromagnetic communication is the most widely used communication. In underwater, electromagnetic radiation gets attenuated. They do not propagate well in underwater. Optical radiation also gets attenuated in underwater scenario. Light is strongly scattered and absorbed underwater, though blue-green wavelengths may be used for short-range, high-bandwidth connections in extremely clear water. [6]. As there exist a vast difference between electromagnetic communication and underwater communication, networking protocols for underwater communication differ significantly from that of terrestrial communication. However no widely accepted framework exists for the underwater sensor networks[4].

It is the physical channel that makes UWSN unique. Up to today, the only practical solution for underwater communication with acceptable range is utilizing acoustic signal, which travels underwater with longer distance, less attenuation, and higher reliability. Hence acoustics play a significant role in underwater communication. Hydrophones are used to transmit acoustic power and allow to receive the acoustic power impinging on it.
Generally the hydrophones are made up of piezoelectric materials like PZT (Lead Zirconium Titanate). Main specifications for underwater acoustic transducers are power carrying capability, the depth of operation and sensitivity of the transducer.

The problems with the acoustic communication are low speed, dependence on the temperature profile of the day, attenuation, multi path propagation and less bandwidth. Underwater acoustic communications are mainly influenced by path loss, noise, multi-path, Doppler spread, and high and variable propagation delay. All these factors determine the temporal and spatial variability of the acoustic channel, and make the available bandwidth of the Underwater Acoustic channel limited and dramatically dependent on both range and frequency. Long-range systems that operate over several tens of kilometers may have a bandwidth of only a few $\mathrm{kHz}$, while a short-range system operating over several tens of meters may have more than a hundred $\mathrm{kHz}$ of bandwidth. In both cases these factors lead to low bit rate, in the order of tens of kbit/s for existing devices.

Fabrication of an acoustic modem with a rugged pressure housing is complex. The other supporting devices like an underwater connector and an underwater cable also increases the cost of the acoustic modem[2]. Commercially available acoustic modems operate in the region of 16 to $30 \mathrm{KHz}$. The range of the commercial modems will depend on the bathy profile of the day, sea state, type of sea bottom and depth of the water.

\section{ARCHITECTURE OF AN UNDERWATER SENSOR NETWORK}

Architecture of Underwater Sensor Network (UWSN) has been discussed in [1].These networks consist of static sensor nodes and Autonomous underwater vehicles. Static sensor nodes are immovable sensor nodes which work from a fixed location. These nodes will be either anchored to the sea bottom or will be floating using a flotation device which keeps the assembly upright on the sea floor

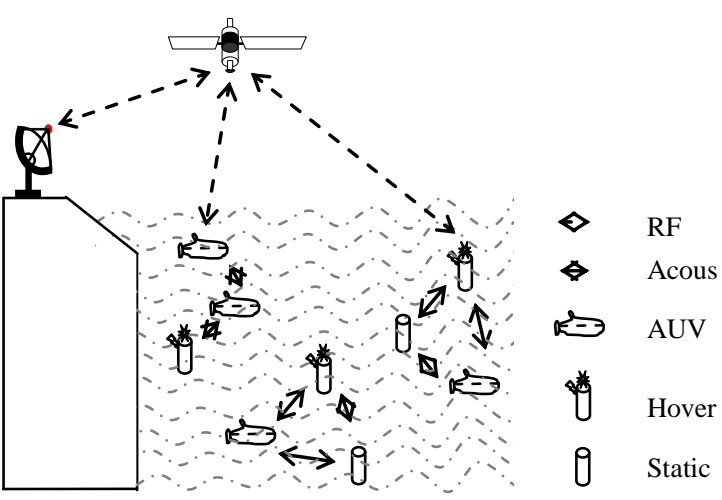

Figure 1: Proposed network architecture

Autonomous Underwater Vehicles are unmanned vehicles which can be preprogrammed for certain trajectories and for specific missions. Applications are in diverse fields in civil and military sectors. AUVs can be classified into heavy weight AUV's, light weight AUV's and micro AUVs. The mechanical console of an AUV has to 
withstand the water pressure for which it is aimed. The power source of the fixed node as well as the AUV is normally a battery with adequate capacity to energize the onboard electronics and sensors of the nodes for periods of long endurance. Acoustic communication also demands more power. The sensor node has to be operational for a few days at least, as frequent access for any maintenance is a complex exercise. All these make the power source a quite critical system of the network. Sensors namely pressure transducer, paddle wheel speed sensor, MEMs are used in the sensor node for measuring the parameters.

Concept of hovering sensor nodes have been introduced in this research work. These nodes will have almost neutral buoyancy and can hover at a particular location. Hence they will be able to stay at a fixed depth, if the effects of currents are less. These sensor nodes will be able to move up and down using a small motor. The motor will drive the propellers. The propellers can move either in clock wise or anti clockwise direction. The direction will be controlled by the on board computer unit. Hovering also enables the sensor nodes to acquire the GPS data by coming to surface

Several kinds of sensors can be employed for surveillance, usually a combination of acoustic, magnetic and pressure sensors[5].When a platform made of ferrous material moves, there will be a small magnetic anomaly. A threeaxis fluxgate magnetic sensor which can detect small magnetic anomalies of the order of tens of micro tesla can be employed to detect the presence of any platform.

\section{DEVELOPMENT OF A STATIC UNDERWATER SENSOR NODE}

Static Underwater sensor Node is useful in the acquisition of data in a remote place. It is an important constituent of an Underwater Sensor Network. The node is powered on when it senses the presence of water using the water contact switch. The sensor node floats on the surface of the water. Then it can communicate with the other nodes using the acoustic modem and it can communicate to the command centre using the satellite transmitter. GPS receiver on board the unit helps to gets its own localisation information.

The main parts of this sensor node are

$$
\begin{aligned}
& \text { 1. Mechanical Console } \\
& \text { 2. Embedded Computer Module } \\
& \text { 3. Communication Module } \\
& \text { 4. Acoustic Modem } \\
& \text { 5. Battery Section }
\end{aligned}
$$

It is an embedded Computer based compact, rugged and waterproof system designed for under water application. Sensor Node consists of console-top cover and bottom body to house the electronics. Compact GPS receiver with patch antenna is present at the top, which can locate its position through Satellite. System also consists of S-band transmitting antenna with power amplifier to transmit GPS parameters and other critical parameters to satellite.

System consists of embedded computer for GPS acquisition, transmission message scheduling, IR data reception and implementation of other logics. It has high power rating rechargeable batteries for long time operations. It also has on board flash memory to store system parameters and records. ADC is present to know the status of internal battery.

\subsection{Mechanical console}

The underwater node has a water tight console. The present assembly is indented for operation at external hydrostatic pressure of 500 meters. Assembly is made of Perspex material that can sustain 50 bar of hydrostatic pressure. The system is basically positive buoyant which makes it to float in water. By adding the weight, the system can be made negative buoyant. Perspective view of the network node is shown figure. All the electronic modules and battery has been assembled in the mechanical console.

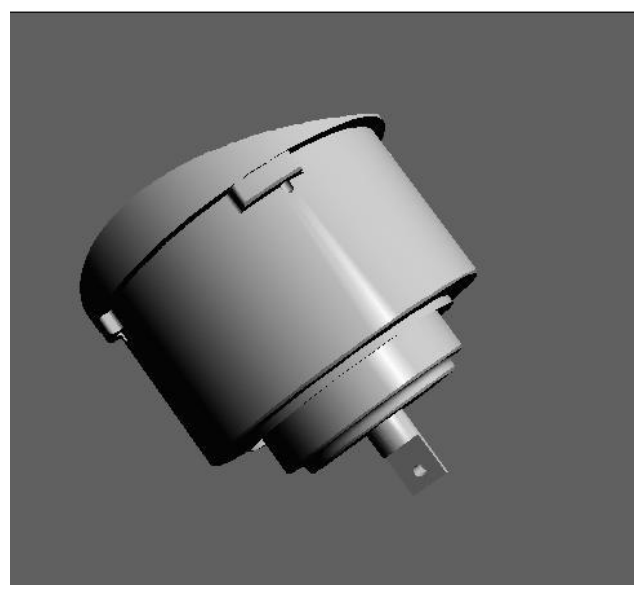

Figure 2: Perspective view of the sensor node

\subsection{Embedded Computer Module}

The embedded computer module is the central unit of under water node. It controls and sequences the operation of the other modules. It also receives the NMEA data from the GPS chip and transmits the data to the $\mathrm{S}$ band transmitter.

\subsection{Communication Module}

The communication terminal of node transmits its current GPS position at 300bps. The node has a built-in GPS receiver from which the current position of the terminal is fetched. The communication terminal transmits the GPS information in S-band to satellite, which in-turn, is sent to the ground based receiving station in $\mathrm{C}$ - band. In the receiving station, the $\mathrm{C}$-band signal is down converted and demodulated for extraction of transmitted GPS data. This enables continuous monitoring of location of the sensor node. GPS receiver Navsys make GE-370 has been selected for this purpose. It is a low power and high sensitive, 20 channels GPS receiver. The satellite transmitter has a power of $8 \mathrm{w}$ to account for the losses due to the sea water splashing. Antenna section consists of Sband antenna and GPS antenna.

\subsection{Acoustic Modem}

Acoustic modem provides the means of communication between the network nodes. As explained earlier acoustic communication is the only method of exchanging data in an underwater network. Here we have used an off the shelf standard acoustic modem of make M/s Dspcomm. The input to the acoustic modem is RS 232 data. The modem can receive and transmit data in different bit rates from $300 \mathrm{bps}$ to $2400 \mathrm{bps}$. 


\subsection{Embedded Software of the node}

The software on board the underwater node has various modules namely the GPS receiver module, Infra Red communication module, satellite communication module and acoustic communication module. The scheduler of the software is derived from a10 millisecond timer interrupt. All the timings are derived from this basic timer.

\subsection{Testing Scheme}

The embedded software as per the requirements have been developed, compiled and flashed to the embedded computer board. Embedded computer board has been assembled and integrated with the mechanical console. A test set up has been made to test the functionalities of the underwater node. Test system simulates the interface signals namely the water contact input, and the GPS simulation. The prototype network node has been assembled, tested in lab with the test setup. Subsequently the node has been subjected to field trials. The data acquisition during the underwater phase and data reception from the test system has been verified and found satisfactory. The GPS acquisition and transmission of the localization data also has been verified.

\section{CONCLUSION}

Realizing an underwater acoustic network, is a difficult task, this paper reviews the challenges and issues of underwater acoustic sensor networks. Further it discusses the constituents of the network. This paper also has dealt with the development of a node for the under water network.

\section{REFERENCES}

[1] I. F. Akyildiz, D. Pompili and T. Melodia, "State-ofthe-art in protocol research for underwater acoustic sensor networks", WUWNet '06: Proceedings of the 1st ACM International Workshop on Underwater Networks, 2006, pp. 7-16.
[2] J. Partan, J. Kurose, and B. N. Levine, " A Survey of Practical Issues in Underwater Networks", ACM International Workshop on UnderWater Networks (WUWNet), pages 17-24, September 2006.

[3] Mandar Chitre, Shiraz Shahabudeen, Milica Stojanovic, "Underwater acoustic communications and networking: Recent advances and future challenges", Marine Technology Society Journal, 2008,Volume 42,Issue 1 103-116.

[4] Zaihan Jiang," Underwater Acoustic Networks Issues and Solutions", International journal of intelligent control and systems, Vol. 13, No. 3, September 2008, 152-161

[5] Lee Freitag, Mathew Grund, Chris Von, "A Shallow Water Acoustic Network For Mine Countermeasures Operations With Autonomous Underwater Vehicles", Underwater Defense Technology (UDT), 2005.

[6] I.Vasilescu, K.Kotay, D.Rus, M.Dunbabin, and P.Corke. "Data collection, storage, and retrieval with an underwater sensor network', SenSys '05, 2005.

[7] J.-H. Cui, J. Kong, M. Gerla, and S. Zhou. Challenges: "Building Scalable Mobile Underwater Wireless Sensor Networks for Aquatic Applications", Special Issue of IEEE Network on Wireless Sensor Networking, May2006.

[8] V.R. Chandrasekhar, W.K.G. Seah, Y S. Choo, H.V. $\mathrm{Ee}$, "Localization in underwater sensor networks survey and challenges", Proceedings of the First ACM International Workshop on Under-Water Networks (WUWNet), in conjunction with ACM MobiCom 2006, Los Angeles, California, USA September 2006. 